\title{
ESTUDIO COMPARATIVO DE LA EXPRESIÓN DE p53, Ki67, bcl-2 y CK20 EN EL CARCINOMA TRANSICIONAL SUPERFICIAL DE VEJIGA: CORRELACIÓN CON LA RECURRENCIA, GRADO HISTOLÓGICO Y ESTADIO CLÍNICO
}

\author{
P. SAN MIGUEL FRAILE, I. ANTÓN BADIOLA, J.A. ORTIZ REY, C. ÁLVAREZ ÁLVAREZ,
} A. FERNÁNDEZ COSTAS, M. LAGO FERNÁNDEZ, E. PELAEZ BOISMORAND, E. ZUNGRI TELO*, A. DE LA FUENTE BUCETA

Servicio de Anatomía Patológica. *Servicio de Urología. Centro Médico POVISA. Vigo. Pontevedra.

Actas Urol Esp. 27 (8): 587-593, 2003

\section{RESUMEN}

ESTUDIO COMPARATIVO DE LA EXPRESIÓN DE p53, Ki67, bcl-2 y CK20 EN EL CARCINOMA TRANSICIONAL SUPERFICIAL DE VEJIGA: CORRELACIÓN CON LA RECURRENCIA, GRADO HISTOLÓGICO Y ESTADIO CLÍNICO

OBJETIVO: Analizar la correlación entre la expresión de p53, Ki67, bcl-2 y CK20, detectadas por técnicas de inmunohistoquímica, con las variables pronósticas clásicas: grado histológico, estadio clínico y recurrencia del tumor.

MATERIAL Y MÉTODOS: Estudiamos 57 biopsias de carcinomas transicionales superficiales de vejiga. Las biopsias valoradas correspondian a 36 y 21 carcinomas transicionales de vejiga no recidivantes y recidivantes, respectivamente. Determinados la correlación entre la expresión de p53, Ki-67, bcl-2 y CK20 con el estadio clínico, grado histológico y la recurrencia del tumor.

RESULTADOS: Demostramos una correlación estadísticamente significativa entre la expresión de Ki-67 y p53 con el grado histológico y la recidiva del tumor. No demostramos asociación estadísticamente significativa entre la expresión de bcl-2 y CK20 con el grado histológico, estadio clínico y recurrencia del tumor.

CONCLUSIONES: La expresión de p53 y Ki-67 aumenta conforme aumenta el grado histológico del tumor y permite predecir la recurrencia de los carcinomas transicionales superficiales de vejiga.

PALABRAS CLAVE: Carcinoma transicional superficial. Vejiga. Proteína p53. Ki-67. bcl-2. CK20.

\section{ABSTRACT}

COMPARATIVE ANALYSIS OF p53, Ki-67, bcl-2 AND CK20 EXPRESSION IN SUPERFICIAL TRANSITIONAL CELL CARCINOMA OF URINARY BLADDER: CORRELATION WITH RECURRENCE, HISTOLOGICAL GRADE AND CLINICAL STAGE

OBJETIVE: We examined the presence of p53, Ki-67, bcl-2 and CK20, as detected by immunohistochemistry, and correlated with the classic variables (grade, stage and recurrence).

MATERIAL AND METHOD: The authors evaluated 57 superficial transitional cell carcinomas. Biopsy specimens examined included non recurrent transitional cell carcinomas $(n=36)$ and recurrent transitional cell carcinomas $(n=21)$. Association of bcl-2, p53, Ki-67 y CK20 index immunoreactivity with tumor grade, clinical stage and tumor recurrence was examined.

RESULTS: Ki-67 and p53 expression were related to the degree of differentiation and recurrence of the disease. bcl-2 and CK20 were not correlated with grade, stage and recurrence of the disease.

CONCLUSIONS: Positivity for Ki-67 and p53 increase with grade of the disease. P53 and Ki-67 are predictors of tumor recurrence for patients with superficial transitional cell carcinoma.

KEY WORKS: Transitional cell carcinoma. Bladder. p53 protein. Ki-67. bcl-2. CK20. 
$\mathrm{E}_{\mathrm{p}}^{\mathrm{sit}}$ stá ampliamente aceptado que la mayor parte de las enfermedades neoplásicas se caracterizan por una proliferación incontrolada y por su pérdida de diferenciación celular ${ }^{1}$. Además de estas alteraciones las células cancerosas adquieren durante el proceso de transformación maligna, capacidad para infiltrar, metastatizar, así como resistencia a múltiples fármacos. Estas características son reflejo de las alteraciones genéticas que puede ser debidas básicamente a activación de proto-oncogenes o inactivación de genes supresores.

Tradicionalmente, se consideró que los factores pronósticos más importantes en el carcinoma transicional de vejiga eran el grado histológico y el estadio clínico ${ }^{2}$. Sin embargo, actualmente se sabe que dos tumores morfológicamente similares pueden tener una agresividad biológica completamente diferente ${ }^{3,4}$. Por este motivo, se están incorporando nuevos marcadores inmunohistoquímicos capaces de detectar alteraciones genéticas que nos permitan predecir la agresividad y/o recurrencia de estos tumores y así poder diseñar nuevas estrategias terapéuticas.

En los últimos años se ha desvelado que la función de algunos protoncogenes como el bcl-2 $2^{2,5-10}$, genes supresores como el p53 $3^{2,6,11-16}$, factores de proliferación celular como el Ki672,4,5,10,17-23 y la expresión de ciertas queratinas como CK20 $24-26$ podrían estar relacionados con el grado histológico, estadio clínico y la recurrencia del carcinoma transicional de vejiga. Numerosos estudios han evaluado el grado de expresión de algunos de estos marcadores inmunohistoquímicos en el carcinoma transicional de vejiga con resultados controverti$\operatorname{dos}^{2,4-19}$.

El objetivo de este estudio es valorar la expresión de p53, Ki-67, bcl-2 y CK20 en resecciones transuretrales con carcinomas transicionales superficiales de vejiga (estadio pTa y pT1) y estudiar la correlación de estos parámetros inmunohistoquímicos con la recurrencia, grado histológico y estadio clínico.

\section{MATERIAL Y MÉTODOS}

Hemos estudiado retrospectivamente 57 biopsias de vejiga tomadas al azar del archivo de nuestro servicio y que fueron diagnosticadas en nuestro Servicio de carcinoma transicional de vejiga entre los años 1987-1996. Treinta y seis biopsias corresponden a carcinomas transicionales superficiales de vejiga no recurrentes y 21 biopsias a carcinomas transicionales de vejiga recurrentes. En los tumores recurrentes el estudio inmunohistoquímico se realizó en la primera biopsia. El período de seguimiento mínimo en los carcinomas transicionales fue de 6 años. En todos los casos se revisaron las biopsias para confirmación del diagnóstico histológico.

Para la inmunohistoquímica se utilizaron los anticuerpos monoclonales p53, Ki-67, bcl-2 y CK20, cuyo fabricante, dilución de trabajo y designación del clon viene especificado en la Tabla I. En la p53, Ki-67 y bcl-2 la valoración se hizo cuantitativamente, expresando el resultado en porcentajes. Para la CK20 la valoración fue cualitativa: positiva o negativa.

La técnica de inmunohistoquímica se realizó sobre secciones tratadas previamente a la inmunotinción para facilitar el acceso de los anticuerpos a los antígenos tisulares. Se utilizó la olla a presión durante 5 minutos en tampón citrato a $\mathrm{pH}^{28}$. Posteriormente se realizó una inhibición de la peroxidasa endógena con peróxido de hidrógeno al 3\% durante 10 minutos y se incubaron con el anticuerpo primario diluido adecuadamente en tampón tris-salino durante un tiempo variable según el anticuerpo empleado. El método utilizado para la detección fue el de estreptoavidinabiotina marcada con peroxidasa y el cromógeno del revelado fue diaminobencidina (DAB).

Antes de proceder a la cuantificación de los resultados de inmunohistoquímica, se valoró en todos los casos la calidad de la técnica de inmunohistoquímica y se seleccionaron las áreas con más positividad, evitando mediciones en áreas periféricas, zonas desvitalizadas, con necrosis o artefacto.

\section{TABLA I}

CARACTERÍSTICAS DE LA TÉCNICA

DE INMUNOHISTOQUÍMICA PARA LOS DISTINTOS ANTICUERPOS

\begin{tabular}{||l|c|c|c|c|}
\hline Anticuerpo & Clon & Dilución & Procedencia & $\begin{array}{c}\text { Tiempo de } \\
\text { incubación }\end{array}$ \\
\hline P53 & DO-7 & $1: 100$ & Dako ${ }^{\circledR}$ & 30 minutos \\
\hline Ki67 & Ki S5 & $1: 50$ & Dako ${ }^{\circ}$ & 1 hora \\
\hline Bcl-2 & 124 & $1: 80$ & Dako ${ }^{\circledR}$ & 30 minutos \\
\hline CK20 & Ks 20,8 & $1: 25$ & Dako ${ }^{\circledR}$ & 30 minutos \\
\hline
\end{tabular}


En el estudio estadístico se incluyen las siguientes variables: grado histológico (G1, G2 o G3), estadio clínico (pTa o pT1) y recurrencia (sí o no) con la expresión de los distintos marcadores inmunohistoquímicos: p53, Ki 67, bcl-2 y CK20. El estudio estadístico se analizó mediante el test de $\mathrm{X}^{2}$ o la prueba exacta de Fisher. Se consideró que un valor era estadísticamente significativo, cuando $\mathrm{p} \leq 0,05$.

\section{RESULTADOS}

Las características clínicopatológicas se muestran en la Tabla II. La edad de los enfermos osciló entre los 46 y 86 años (media 70 años). El seguimiento clínico está comprendido entre los 180 y 72 meses (media de 156 y 108 meses para los tumores no recurrentes y recurrentes, respectivamente).

La expresión en porcentajes de los distintos marcadores inmunohistoquímicos según grado histológico, estadio clínico y recurrencia del tumor está reflejada en las Tablas III, IV y V.

$\mathrm{El}$ estudio estadístico entre las tres variables analizadas (grado histológico, estadio clínico y recurrencia) con los distintos parámetros inmunohistoquímicos se refleja en la Tabla VI. Los parámetros inmunohistoquímicos relacionados con el grado histológico fueron el índice de proliferación celular: Ki-67 (p=0,003) y p53 (p=0,043), apreciándose un incremento de la actividad proliferativa y de la expresión de p53, conforme aumenta el grado histológico del tumor.

\section{TABLA II}

DATOS CLÍNICO-PATOLÓGICOS DE LOS CARCINOMAS TRANSICIONALES SUPERFICIALES DE VEJIGA

\begin{tabular}{||l|c|c||}
\hline \multirow{2}{*}{} & \multicolumn{2}{|c|}{$N^{\circ}$ de casos } \\
\cline { 2 - 3 } & $\begin{array}{c}\text { Recurrente } \\
(n=21)\end{array}$ & $\begin{array}{c}\text { No recurrente } \\
(n=36)\end{array}$ \\
\hline Edad: & 70 & 69 \\
\hline Sexo: & & \\
$\bullet$ Hombres & $16(76 \%)$ & $32(89 \%)$ \\
$\bullet$ Mujeres & $5(24 \%)$ & $4(11 \%)$ \\
\hline Grado histológico: & $8(38 \%)$ & $11(31 \%)$ \\
$\bullet$ G1 & $10(48 \%)$ & $16(44 \%)$ \\
$\bullet$ G2 & $3(14 \%)$ & $9(25 \%)$ \\
$\bullet$ G3 & $13(62 \%)$ & $21(58 \%)$ \\
\hline Estado clínico: & $8(38 \%)$ & $15(42 \%)$ \\
\hline PTa & & \\
\hline
\end{tabular}

TABLA III

PORCENTAJE DE LOS DISTINTOS MARCADORES INMUNOHISTOQUÍMICOS SEGÚN EL GRADO HISTOLÓGICO

\begin{tabular}{|l|c|c|c||}
\hline & $\begin{array}{c}\text { Grado I } \\
(n=19)\end{array}$ & $\begin{array}{c}\text { Grado II } \\
(n=26)\end{array}$ & $\begin{array}{c}\text { Grado III } \\
(n=12)\end{array}$ \\
\hline P53 & $27 \%$ & $40 \%$ & $41 \%$ \\
\hline Ki67 & $9 \%$ & $20 \%$ & $30 \%$ \\
\hline Bcl-2 & $6 \%$ & $2 \%$ & $1 \%$ \\
\hline CK20 & $10+/ 9-$ & $7+/ 19-$ & $6+/ 6-$ \\
\hline
\end{tabular}

\section{TABLA IV}

PORCENTAJE DE LOS DISTINTOS MARCADORES INMUNOHISTOQUÍMICOS SEGÚN EL ESTADIO CLÍNICO

\begin{tabular}{|l|c|c|}
\hline & $\begin{array}{c}P T a \\
(n=34)\end{array}$ & $\begin{array}{c}p T 1 \\
(n=23)\end{array}$ \\
\hline P53 & $30,6 \%$ & $43,4 \%$ \\
\hline Ki67 & $13,7 \%$ & $29,6 \%$ \\
\hline Bcl-2 & $3,8 \%$ & $2,1 \%$ \\
\hline CK20 & $15+/ 19-$ & $8+/ 15-$ \\
\hline
\end{tabular}

TABLA V

PORCENTAJE DE LOS DISTINTOS MARCADORES INMUNOHISTOQUÍMICOS SEGÚN LA RECURRENCIA

\begin{tabular}{||l|c|c||}
\hline \hline p53 & $\begin{array}{c}\text { Recurrentes } \\
(n=21)\end{array}$ & $\begin{array}{c}\text { No recurrentes } \\
(n=36)\end{array}$ \\
\hline Ki67 & $52 \%$ & $29,4 \%$ \\
\hline Bcl-2 & $18,19 \%$ & $20,4 \%$ \\
\hline CK20 & $3,3 \%$ & $3,5 \%$ \\
\hline
\end{tabular}

Ninguno de los factores estudiados demostraron asociación estadística con el estadio clínico del tumor. Por último cuando se estudió la asociación estadística entre los distintos parámetros inmunohistoquímicos y la recurrencia del tumor, se demostró una mayor expresión de p53 en los tumores recurrentes (Tabla VI).

\section{DISCUSIÓN}

El $80 \%$ de los carcinomas transicionales de vejiga son superficiales ${ }^{6} \mathrm{y}$ aproximadamente el $70 \%$ recidivan después de la extirpación ${ }^{2}$. Tradicionalmente se ha considerado que el grado histológico y el estadio clínico eran los factores pronósticos más importantes en el carcinoma 


\section{TABLA VI}

CARCINOMA TRANSICIONAL SUPERFICIAL DE VEJIGA RECURRENTE Y NO RECURRENTE. RESULTADOS DE ANÁLISIS ESTADÍSTICO DEL GRADO HISTOLÓGICO Y ESTADIO CLÍNICO CON LOS PARÁMETROS INMUNOHISTOQUÍMICOS

\begin{tabular}{||l|c|c|c||}
\hline & Grado histológico & Estadio clínico & Recurrencia \\
\hline P53 & $\mathrm{p}=0,04$ & $\mathrm{p}=0,23$ & $\mathrm{p}=0,03$ \\
\hline Ki-67 & $\mathrm{p}=0,003$ & $\mathrm{p}=0,30$ & $\mathrm{p}=0,65$ \\
\hline Bcl-2 & $\mathrm{p}=0,49$ & $\mathrm{p}=0,89$ & $\mathrm{p}=0,09$ \\
\hline CK20 & $\mathrm{p}=0,66$ & $\mathrm{p}=0,49$ & $\mathrm{p}=0,56$ \\
\hline
\end{tabular}

transicional superficial de vejiga $^{2}$ pero en muchas ocasiones no es posible predecir la recurrencia de los mismos basados en estos criterios, como se demuestra en nuestro trabajo y otros similares ${ }^{3,4}$.

Muchos estudios han valorado la asociación de distintos marcadores inmunohistoquímicos como bcl-2 $2^{2,5-10}$, p53 $3^{2,6,11-17}$, Ki $67^{5,10,18-24}$ y CK20 ${ }^{25-27}$ con los factores pronósticos clásicos y con la recurrencia pero los resultados son contradictorios y las series difíciles de comparar, principalmente por la gran heterogeneidad en el estadio clínicos de los diferentes trabajos.

Nosotros hemos analizado la utilidad de estos marcadores inmunohistoquímicos en los carcinomas transicionales de vejiga, y lo hemos correlacionado con las variables pronósticas clásicas (estadio clínico y grado histológico) y la recurrencia del tumor.

La expresión media de bcl-2 en los carcinomas transicionales superficiales de vejiga de nuestra serie fue del 3,4\%. Estos resultados son llamativamente inferiores a los publicados en la literatura, cuya variabilidad oscila entre el $10 \mathrm{y}$ el $70 \%^{5-10}$. Su expresión, aunque variable, es casi constante en las capas basales del epitelio transicional sano ${ }^{6}$, y suele estar disminuida en el carcinoma transicional de alto grado y estadio clíni$\mathrm{co}^{5,10}$. Algunos autores consideran que la expresión de bc-2 podría tener un efecto "protector" en estas neoplasias ${ }^{5}$, como ya se ha demostrado en tumores de otra localización ${ }^{29-31}$.

Con respecto a la asociación de la expresión de bcl-2 con el grado histológico y estadio clínico se aprecian marcadas variaciones según las distintas series ${ }^{4,6-10}$. Nuestros resultados se mues- tran acordes con los presentados por Nakopoulou y cols. ${ }^{5}$, ya que apreciamos una relación inversa entre el grado histológico y el estadio del tumor, aunque sin demostrar una asociación estadística significativa.

Por último, en nuestro estudio y coincidiendo con Wu y cols. ${ }^{4}$, la expresión de bcl-2 no tuvo capacidad predictiva en la recurrencia del tumor. Los resultados en la literatura son variables y contradictorios ya que mientras Atug ${ }^{8}$ y Kirsh $^{9}$ consideran que la expresión de bcl-2 tiene un pronóstico adverso, Vollmer ${ }^{2}, \mathrm{Li}^{6}$ y Lipponen $^{7}$ consideran que tienen un efecto protector. A nuestro entender, estos resultados deben de ser confirmados mediante estudios en series más amplias.

Las mutaciones de p53 son muy frecuentes en los cánceres humanos, reflejando una proliferación incontrolada y una pérdida de diferenciación celular. El urotelio benigno generalmente no muestra tinción positiva para la p53 ${ }^{32}$, mientras que la expresión en el carcinoma transicional de vejiga varía entre el $17-78 \%^{2,5,6,12,13,33-36}$. En nuestra experiencia la expresión media de p53 fue de $36 \%$. Esta gran variabilidad en los resultados puede ser reflejo de la gran heterogeneidad regional ${ }^{32}$, los distintos puntos de corte empleados para determinar si la tinción es positiva o negativa $^{36}$ y por último los diferentes métodos para cuantificarlo.

Muchos estudios han verificado la relación existente entre la expresión de p53 con el grado histológico, estadio clínico y recurrencia del tumor ${ }^{5,15,17,37-39}$. Estos datos sugieren la posibilidad de que la expresión de p53 sea un marcador de progresión y agresividad tumoral, apoyándose precisamente en la escasa o nula expresión de este marcador en el urotelio normal. Nuestro trabajo, pone de manifiesto una relación directa significativa entre la expresión de p53 y el grado histológico $(p=0,04)$ y con la recurrencia $(p=0,03)$ del tumor. Sin embargo, no hemos apreciado que la expresión de p53 guarde relación estadísticamente significativa con el estadio clínico del tumor, aunque si se aprecia diferencia entre los tumores estadio pTa y pT1.

En nuestro trabajo se aprecia como el porcentaje medio de expresión de p53 es mayor en los tumores recurrentes (Tabla V). Cuando valora- 
mos cada caso de forma individual se observaba que algunos tumores no recurrentes tienen una expresión de p53 similar a la de los recurrentes; sin embargo, en los tumores recurrentes la expresión de p53 fue siempre superior al $20 \%$. Estos datos sugieren que la expresión de p53 podría ser un marcador de recurrencia tumoral y pensamos que aquellos tumores que tienen una expresión baja de p53 no suelen recurrir, aunque la expresión alta de p53 no sea sinónimo de recurrencia.

La expresión de Ki-67 en el epitelio transicional sano es bajo y limitado a los estratos más basales ${ }^{18}$. En los carcinomas transicionales superficiales de vejiga la expresión de Ki-67 está aumentada y la distribución es muy heterogénea incluso dentro del mismo tumor. En nuestra serie, el porcentaje medio de expresión de Ki-67 fue del $18 \%$, estos resultados coinciden con la literatura, cuya variabilidad oscila entre el 5 y el $50 \%$, según las diferentes series ${ }^{5}$.

La mayor parte de los autores coinciden en señalar que la expresión de Ki-67 varía significativamente con el grado histológico y estadio clínico del tumor. En nuestro trabajo se aprecia como la expresión de Ki-67 varía de manera significativa respecto al grado histológico. Estos resultados coinciden con gran parte de la literatura, y encontramos diferente expresión de Ki-67 según cada uno de los grados histológicos. El mayor grado de discordancia está en los tumores con grado histológico 2. Posiblemente esto se deba a que es el grupo más heterogéneo y subjetivo para su clasificación y a la distinta proporción según las diversas series.

También apreciamos como la expresión de Ki67 aumenta conforme lo hace el estadio clínico tumoral, pero sin observarse asociación estadística. La mayor parte de los autores coinciden en señalar diferencias entre los tumores superficiales e infiltrantes ${ }^{5,20,23}$ y son menos los estudios que encuentran diferencias dentro de los tumores superficiales ${ }^{19-23}$, como es nuestro caso.

Por último, en nuestra serie no encontramos asociación entre la expresión de Ki-67 y la recurrencia o no del tumor. Los resultados de los diferentes trabajos que analizan la relación entre la expresión de Ki-67 y la recurrencia varían enormemente; esto posiblemente se deba a los dife- rentes sistemas de clasificación, puntos de corte empleados o a anticuerpos monoclonales. Nuestros resultados coinciden con los aportados por Rodríguez et al. ${ }^{17}$, Zlotta et al. ${ }^{40}$ y Pfister et al. ${ }^{41}$, en los cuales la expresión de Ki-67 no parece ser un factor predictivo en la recurrencia.

La influencia de la expresión de CK20 en el carcinoma transicional de vejiga se ha estudiado relativamente poco, y siempre en relación con el diagnóstico ${ }^{39}$, grado histológico ${ }^{40}$ y recurrencia ${ }^{41-43}$. Algunos autores han observado que la expresión de CK20 puede ser usada como marcador de recurrencia en el carcinoma transicional ${ }^{42}$. Otro estudio más reciente, demostró una mayor tasa de recurrencia en los tumores transicionales con expresión anormal de $\mathrm{CK} 2 \mathrm{O}^{43}$, pero sin demostrar asociación estadística, por lo que los resultados no son consistentes.

En nuestro estudio la $\mathrm{Ck} 20$ fue positiva en el $40 \%$ de los casos y no encontramos buena correlación de la expresión de CK20 con la recurrencia tumoral, grado histológico ni estadio clínico. Sin embargo, consideramos muy útil el estudio de la expresión de CK20 junto a otras citokeratinas, especialmente CK7, ya que frecuentemente ayuda a determinar el origen de tumores, cuyo primario no es conocido ${ }^{44}$. Su principal aplicación en el aparato genitourinario, es que permite diferenciar el origen vesical o prostático, cuando los carcinomas son pobremente diferenciados.

En resumen, nuestros resultados sugieren que la expresión de p53 puede ser un marcador de recurrencia tumoral en los carcinomas transicionales superficiales de vejiga, por lo que aconsejamos su determinación en los servicios de anatomía patológica, así como un seguimiento clínico más estricto en pacientes con expresión alta de p53.

\section{REFERENCIAS}

1. COHEN SM, ELLWEIN LB.: Cell proliferation and bladder tumor promotion. Prog Clin Biol Res 1991; 369: 347-355.

2. VOLLMER RT, HUMPHREY PA, SWANSON PE et al.: Invasión of the bladder by transitional cell carcinoma: its relation to histologic grade and expresión of p53, MIB-1, cerbB2, epidermal growth factor receptor and bcl-2. Cancer 1998; 82: 715-723.

3. TSUJIHASHI H, NAKANISHI A, MATSUDA H et al.: Cell proliferation of human bladder tumors determined by BrdUrd and Ki-67 immunostaining. J Urol 1991; 145: 846-849. 
4. WU TT, CHEN JH, LEE YX, HUANG JK.: The role of bcl-2, p53 and Ki67 index in predicting tumor recurrence for low grade superficial transitional cell bladder carcinoma. J Urol 2000; 163: 758-760.

5. NAKOPOULOU L, VOURLAKOU C, ZERVAS A, TZONOU A, GAKIOPOULOU H, DIMOPOULOS MA.: The prevalence of bcl-2, p53 and ki67 immunoreactivity in transitional cell bladder carcinomas and their clinicopathologic correlates. Hum Pathol 1998; 29 (2): 146-154.

6. LI B, KANAMARU $\mathrm{H}$, NORIKI S, YAMAGUCHI T, FUKUDA M, OKADA K.: Reciprocal expression of bcl2 and p53 oncoproteins in urothelial dysplasia and carcinoma of the urinary bladder. Urol Res 1998; 26: 235-241.

7. LIPPONEN PK, AALTOMAA S, ESKELINEN M.: Expression of the apoptosis suppressing bcl-2 protein in transitional cell bladder tumours. Histopathology 1996; 28: 135-140.

8. ATUG F, TURKERI L, OZYUREK M et al.: Bcl-2 and p53 overexpression as associated risk factors in transitional cell carcinoma of the bladder. Int Urol Nephrol 1998; 30: 455-461.

9. KIRSH EJ, BAUNOCH DA, STADLER WM. Expression of bcl-2 and bcl-x in bladder cancer. $J$ Urol 1998; 159: 1348-1353.

10. LIUKKONEN TJ, LIPPONEN PK, HELLE M, JAUHIAINEN KE.: Immunoreactivity of bcl-2, p53, and EGFr is associated with tumor stage, grade and cell proliferationin superficial bladder cancer. Finnbladder III Group. Urol Res 1997; 25: 1-7.

11. GLICK SH, HOWELL LP, DEVERE WHITE RW.: Relationship of p53 and bcl-2 to prognosis in muscle-invasive transitional cell carcinoma of the bladder. J Urol 1996; 155: 1754-1757.

12. LIPPONEN PK.: Overexpression of p53 nuclear oncoprotein in transitional cell bladder cancer and its prognostic value. Int I Cancer 1993; 53: 365370.

13. DALBAGNI G, PRESTI JC Jr, REUTER VE et al.: Molecular genetic alterations of chromosome 17 and p53 nuclear overexpression in human bladder cancer. Diag Mol Pathol 1993; 2: 4-13.

14. INAGAKI T, EBISURO S, UEKADO Y et al.: PCNA and p53 in urinary bladder cancer: correlation with histological findings and prognosis. Int $J$ Urol 1997; 4: $172-177$

15. CORDÓN-CARDÓ C, ZHANG ZF, DALBAGNI G et al.: Cooperative effects of p53 and pRB alterations in primary superficial bladder tumors. Cancer Res 1997; 57: 1217-1221.

16. SCHMITZ-DRAGER BJ, KUSHIMA M, GOEBELL P et al.: p53 and MDM2 in the development and progression of bladder cancer. Eur Urol 1997; 32: 487 493.

17. RODRÍGUEZ-ALONSO A, PITA-FERNÁNDEZ S, GONZÁLEZ-CARRERÓ J, NOGUEIRA-MARCH JL.: Multivariate análisis of survival, recurrence, progresión and development of metastasis in T1 and T2a transicional cell bladder carcinoma. Cancer 2002; 94: 1677-1984.

18. MOYANO CALVO JL, DE MIGUEL RODRIGUEZ M, ORTIZ GAMIZ A et al.: Determinación de ploidía de ADN mediante citometría de flujo, índice Ki-67 y sobre-expresión p53 en 121 carcinomas superficiales de vejiga T1. Estudio retrospectivo correlación con las variables clásicas. Actas Urol Esp 2000; 24 (10): 785-795.

19. STROUOPOULOS EN, IOACKIM-VELOGIANNI E, HASTAZEIS $\mathrm{K}$ et al.: Growth fractions in bladder cancer defined by ki-67 expression, and nuclear organiser regions. J Clin Pathol 1993; 46: 159-165.

20. OKAMURA K, MIYAKE K, KOSHIKAWA T et al.: Growth fractions of transitional cell carcinomas of the bladder defined by the monoclonal antibody ki67. J Urol 1990; 144: 875-878.

21. KRÜGER S, MÜLLER H.: Correlation of morphometry nucleolar organizer regions, proliferating cell nuclear antigen and ki-67 antigen expression with grading and staging in urinary bladder carcinomas. Br J Urol 1995; 75 (4): 480-484.

22. MULDERS AH, VAN HOOTEGEM JC et al.: Prognostic factors as in bladder carcinoma: histological parameters and expression of a cycle-related nuclear antigen Ki-67. J Pathol 1992; 166: 37-43.

23. PFISTER C, BUZELIN F, CVASSE C et al.: Comparative analysis of MIB-1 and p53 expression in human bladder tumours and their correlation with cancer progression. Eur Urol 1998; 33: 278284.

24. LIMAS C, BIGLER A, BAIR R et al.: Proliferative activity of urothelial neoplasms: comparison of BrDU incorporation, Ki-67: association with cancer grade, category and recurrence rate of superficial lesions. Br J Urol 1993; 72: 736-739.

25. CHU P, WU E, WEISS LM.: Cytokeratin 7 and 20 expression in epithelial neoplasms: a survey of 435 cases. Mod Pathol 2000; 13 (9): 962-972.

26. SOUTHGATE J, HARNDEN P, TREJDOSIEWICZ LK.: Cytokeratin expression patterns in normal and malignant urothelium: a review of the biological and diagnostic implications. Histol Histopathol 1999; 14 (2): 657-664.

27. HARNDEN P, MAHMOOD N, SOUTHGATE J.: Expression of cytokeratin 20 redefines urothelial papillomas of the bladder. Lancet 1999; 353: 974 977.

28. CATTORETTI G, BECKER MHG, KEY G et al.: Monoclonal antibodies organist recombinant parts of the ki-67 antigen detected proliferatig cells in microwave-processed formalin-fixed paraffin sections. J Pathol 1992; 168: 357-363.

29. BARTETTON GB, DIEBOLD J, CHRISTOFORIS G et al:: Apoptosis and immunohistochemical bcl-2 expression in colorectal adenomas and carcinomas. Cancer 1996; 77: 255-264.

30. PILOTTI S, COLLINI P, RILKE $\mathrm{F}$ et al.: Bcl-2 protein expression in carcinomas originating from the follicular epithelium of the thyroid gland. $J$ Pathol 1994: 172: 337-342.

31. VERHAEGH MEJM, SANDERS CJG, ARENDS JW et al.: Expression of the apoptosis-suppressing protein bcl-2 in non-melanoma skin cancer. $\mathrm{Br} J$ Dermatol 1995; 132: 740-744.

32. ÁLVAREZ KINDELÁN J, LÓPEZ-BELTRÁN A, REQUENA TAPIA MJ.: Biología molecular en el cáncer vesical. Actas Urol Esp 2000; 24 (8): 604625. 
33. BURKHARD FC, MARKWALDER R, THALMANN GN, STUDER UE.: Inmunohistochemical determination of p53 expression. Urol Res 1997; 25 (Suppl 1): S31-S35.

34. CALISKAN M, TURKERI LN, MANSUROGLU B et al.: Nuclear accumulation of mutant p53 protein: a possible predictor of failure of intravesical therapy in bladder cancer. Br J Urol 1997; 79: 373-377.

35. ESRIG D, ELMAJIAN D, GROSHEN $\mathrm{S}$ et al.: Accumulation of nuclear p53 and tumor progression in bladder cancer. New Engl J Med 1994; 331: 1259-1264.

36. SINIK Z, ALKIBAY T, ATAOGLU O et al.: Correlation of nuclear p53 overexpression with clinical and histopathological features of transitional cell bladder cancer. Int Urol Nephrol 1997; 29: 25-31.

37. YE D, LI H, QUIAN S, SUN Y, ZHENG J, MA Y.: Bcl 2/bax expression and p53 gene status in human bladder cancer: relationship to early recurrence with intravesical quemotherapy after resection. $J$ Urol 1998; 160: 2025-2029.

38. CHANDHARY KS, LU QL, ABEL PD et al.: Expresión of bcl-2 and p53 oncoproteins inschistosomiasis associated transitional and squamous cell carcinoma of urinary bladder. Br J Urol 1997; 79: 78-84.

39. WAGNER U, SAUTER G, MOCH H et al.: Patterns of p53, erb-2 and EGF-r expression in premalignant lesions of the urinary bladder. Hum Pathol 1995; 9: 970-978.

40. ZLOTTA AR, NOEL JC, FAYT I et al.: Correlation and prognostic significance of p53, p2 $1^{\mathrm{WAF} 1 / \mathrm{CIP} 1}$ and Ki-67 expression in patients with superficial bladder tumors treated with Bacillus CalmetteGuérin intravesical therapy. J Urol 1999; 161: 792798.

41. PFISTER C, LACOMBE L, VEZINA MC et al. Prognostic value of the proliferative index determined by Ki-67 immunostaining in superficial bladder tumors. Hum Pathol 1999; 30 (11): 1350-1355.

42. HARNEN P; MAHMOOD N; SOUTHGATE J.: Expression of cytokeratin 20 redefines urothelial papillomas of the bladder. Lancet 1990: 353 (20): 974-977.

43. ALSHEIKH A, MOHAMEDALI $Z$, JONES E, MASTERSON J, GILKS CB.: Comparison of the WHO/ISUP classification and cytokeratin 20 expression in predicting the behaviour of low-grade papillary urothelial tumors. World/Health Organization /International Society of Urologic Pathology. Mod Pathol 2002; 14 (4): 267-272.

44. CID P, ORTIZ-REY JA, ANTÓN I et al.: Expresión coordinada de CK7 y CK20 en el carcinoma transicional de vejiga: utilidad diagnóstica. Actas Urol Esp 2002; 26: 279-284.

\section{Dra. P. San Miguel Fraile \\ Centro Médico POVISA \\ C/ Salamanca, 5 \\ 36211 Vigo (Pontevedra)}

(Trabajo recibido el 18 diciembre de 2002) 